\title{
NUEVOS REGISTROS EXTIENDEN DISTRIBUCIONES DE TRES ESPECIES DE HYMENOPHYLLACEAE (PTERIDOPHYTA) A LA REGION DEL MAULE, CHILE
}

\author{
NEW RECORDS EXTEND DISTRIBUTIONS OF THREE SPECIES OF \\ HYMENOPHYLLACEAE (PTERIDOPHYTA) TO THE MAULE REGION, \\ CHILE
}

This article reports the occurrence of three species of Hymenophyllaceae in the coast range of the Maule region in central Chile. The discovery of Hymenoglossum cruentum (Cav.) K. Presl., Hymenophyllum darwinii Hook.f. ex Bosch y Trichomanes exsectum Kunze at the Fundo San Pedro/ Las Cañas $\left(35^{\circ} 29^{\prime} \mathrm{S}-72^{\circ} 22^{\prime} \mathrm{W}\right)$ represents sizeable extensions of the known ranges of all three ferns, which are mainly associated with humid temperate forests further south. These findings provide further evidence that humid gully habitats in the Mediterranean climate of central Chile harbour many elements of the temperate rainforests which currently dominate landscapes further south.

\section{Alexandra Stoll ${ }^{1} \&$ Steffen Hahn ${ }^{2}$}

\begin{abstract}
${ }^{1}$ Technische Universität Dresden, Fakultät für Mathematik und Naturwissenschaften, Fachrichtung Biologie, Institut für Botanik, 01069 Dresden, Alemania, e-mail: alexandrastoll@hotmail.com ${ }^{2}$ Jardín Botánico Universidad de Talca, Casilla 747, Talca, Chile, e-mail: mexhahn@gmx.de
\end{abstract}

En Chile los helechos de la familia Hymenophyllaceae están representados por 24 especies repartidas en cuatro géneros. Se distribuyen principalmente en la zona templada del país, en zonas con precipitaciones mínimas anuales de 2000 $\mathrm{mm}$ y con un patrón de clima marítimo. En regiones más septentrionales, con climas menos húmedos, se refugian en hábitats con carácter micro-climático, como quebradas con abundante vegetación o cuevas (Diem \& Lichtenstein 1959).

Este artículo informa por primera vez la presencia de tres especies de Hymenophyllaceae en la Cordillera de la Costa de la VII Región de Chile. Los nuevos registros de Hymenoglossum cruentum (Cav.) K.Presl., Hymenophyllum darwinii Hook.f. ex Bosch y Trichomanes exsectum Kunze constituyen una importante extensión de rango para cada una de las tres especies, estableciendo nuevos límites septentrionales en Chile continental y una presencia que, por ahora, puede ser considerada relictual.

Estos registros agregan nuevos elementos a la discusión sobre la fitogeografia histórica en el área costera del centro-sur de Chile (Villagrán et al. 1998) y realzan la significancia de la flora regional. Esta información viene a sumarse a varios otros informes de la presencia de especies propias de los bosques lluviosos en la Cordillera de la Costa de la VII Región (Troncoso \& San Martín 1988; Sepúlveda \& Stoll 2003; Sepúlveda \& Troncoso 2004).

Las populaciones fueron encontradas en dos quebradas del fundo San Pedro/Las Cañas, comuna de Empedrado, ubicado en la cordillera costera de la Región del Maule. Ambas drenan cuerpos fluviales locales durante todo el año. Las principales especies leñosas de la vegetación en el fondo de los valles son principalmente elementos valdivianos, como Nothofagus dombeyi (Mirb.) Oerst., Podocarpus saligna D. Don, Saxegothaea conspicua Lindl., Weinmannia trichosperma Cav., Desfontainea spinosa Ruiz et. Pav., Gevuina avellana Molina, Tepualia stipularis (Hook. et Arn.) Griseb. y Lophosoria quadripinnata (J.F. Gmel.) C. Chr., acompañados por las trepadoras Mitraria coccinea Cav. y Sarmienta repens Ruiz et Pav.

La quebrada Pellín se ubica en el sureste del fundo (Fig. 1). Es parte de un área de conservación 
establecida por la empresa Forestal Copihue S.A. llamada Parque "Los Hualos de Loanco". Se protege un fragmento de aproximadamente 690 ha de bosque nativo. La vegetación presenta un nivel muy bajo de antropización.

La quebrada Los Coigües se encuentra más al noroeste (Fig. 1). Hacia el borde superior del valle la vegetación natural fue sustituida por plantaciones de Pinus radiata D. Don. La vegetación natural en el fondo del valle se ha podido mantener, a pesar de que se registran individuos asilvestrados de Pinus radiata y la presencia de otras neófitas, como Rubus ulmifolius Schott o Genista monspessulana (L.) O. Bolòs et Vigo.

\section{Hymenoglossum cruentum (Cav.) K. Presl.}

El género Hymenoglossum K. Presl. es monotípico y endémico en Chile. La distribución actual de $H$. cruentum en Chile se extiende principalmente en la cordillera costera entre la provincia de Arauco y la provincia de Ultima Esperanza (37³6' a 50²9' S), además del Archipiélago de Juan Fernández. Su estado de conservación es "vulnerable", debido a fuertes intervenciones humanas en su hábitat (Rodríguez 1995, Baeza et al. 1998).

El hallazgo de $H$. cruentum ocurrió en la quebrada Pellín, afluente al río Pinotalca (35²9' $\mathrm{S}$ $72^{\circ} 21^{\prime} \mathrm{W}$ ) a una altitud de $350 \mathrm{snm}$ (Fig. 1). Esto implica una ampliación del área de aproximadamente $230 \mathrm{~km}$ al norte del último lugar de registro conocido en Chile continental (37 $36^{\prime} \mathrm{S}-7^{\circ} 21^{\prime} \mathrm{W}$ ).

Esta especie fue encontrada en varias poblaciones distribuidas sobre una distancia de $5 \mathrm{~km}$ a lo largo del curso de agua. Está acompañada por Hymenophyllum fuciforme Sw., Hymeno-phyllum tunbridgense (L.) Sm. y diversas especies de musgos. Crece en las concavidades formadas por rocas sobresalientes con pequeñas filtraciones de agua. A pesar de que algunas plantas mostraban leves signos de desecación estival, se hallaron ejemplares con soros.

Material de estudio: Chile, Séptima Región, provincia de Talca, comuna de Empedrado, quebrada Pellin, $350 \mathrm{~m} \mathrm{snm}, 35^{\circ} 29^{\prime} 51^{\prime}$ ' S y 72 21 '57" W, 13-II-2003, Steffen Hahn y Alexandra Stoll. Herbario de la Universidad de Talca N ${ }^{\circ} 2736$ y 2737.

\section{Hymenophyllum darwinii Hook.f. ex Bosch}

Hymenophyllum darwinii se distribuye tanto en Chile como en Argentina. Según la base de datos del Herbario de la Universidad de Concepción (CONC), su área de distribución en Chile se extiende desde la provincia de Ñuble hasta Tierra del Fuego (36 $34^{\prime}$ a $\left.51^{\circ} 04^{\prime} \mathrm{S}\right)$.

H. darwinii habita en el mismo valle que Hymenoglossum cruentum (Fig. 1). Sin embargo ambas especies no se encontraron creciendo juntas. La ubicación exacta de la población de $H$.darwiniies $35^{\circ} 29^{\prime} \mathrm{S}-72^{\circ} 21^{\prime} \mathrm{W}$, a una altitud de $350 \mathrm{msnm}$. El lugar está situado aproximamente $120 \mathrm{~km}$ al norte del último punto conocido en Chile continental $\left(36^{\circ} 34^{\prime}\right.$ S - $\left.71^{\circ} 58^{\prime} \mathrm{W}\right)$, ampliando así el territorio de $H$. darwinii en dirección septentrional.

Hymenophyllum darwinii se presentó como epífito, creciendo en varios cojines de diferente tamaño sobre los troncos de dos ejemplares Weinmannia trichosperma. Los cojines se hallan junto a varios individuos de Fascicularia bicolor (Ruiz et Pav.) Mez. a una altura entre 2 y $5 \mathrm{~m}$ sobre los fustes. Las láminas de $H$. darwinii demostraban un avanzado nivel de desecación estival, pero no obstante se colectaron ejemplares con soros desarrollados.

Material de estudio: Chile, Séptima Región, provincia de Talca, comuna de Empedrado, quebrada Pellín, 350 m snm, 35²9'S - 72²2’W, 8-XI-2003, Steffen Hahn y Alexandra Stoll. Herbario de la Universidad de Talca $\mathrm{N}^{\circ} 2732$.

\section{Trichomanes exsectum Kunze}

Trichomanes exsectum ha sido mencionado para la X Región desde la provincia de Valdivia a la de Chiloé (3952' a 4306' S) y en el Archipiélago de Juan Fernández (Rodríguez 1995). El helecho crece sobre rocas o epífita en lugares muy sombríos, con preferencia en cavernas (Diem \& Lichtenstein 1959). Su estado de conservación es "rara" (Baeza et al. 1998).

T. exsectum fue colectado en la quebrada Los Coigües $\left(35^{\circ} 27^{\prime} 31^{\prime \prime} \mathrm{S}-72^{\circ} 24^{\prime} 47^{\prime \prime} \mathrm{W}\right)$ a una altitud de 275 msnm (Fig. 1). Esta nueva localidad representa una ampliación considerable del área distribucional, aproximadamente de 490 km más al norte. Crece en una cueva a cuyo costado cae un salto de agua y cuyo piso está inundado. La vegetación 
densa de la quebrada cubre el lado abierto de la cueva. Todo ello mantiene condiciones muy húmedas y muy sombrías. Las plantas habitan encima de la pared rocosa formando una alfombra de al menos $5 \mathrm{~m}^{2}$, conformada por individuos de distintos tamaños $(2,5-18 \mathrm{~cm})$ y estados de desarrollo, incluyendo ejemplares con soros.

Material DE estudio: Chile, Séptima Región, provincia de Talca, comuna de Empedrado, quebrada Los Coigües, $275 \mathrm{~m}$ snm, 35²9'31's 72²2'47'W, 9-XI-2003, Alexandra Stoll y Steffen Hahn. Herbario de la Universidad de Talca N ${ }^{\circ} 2740$.

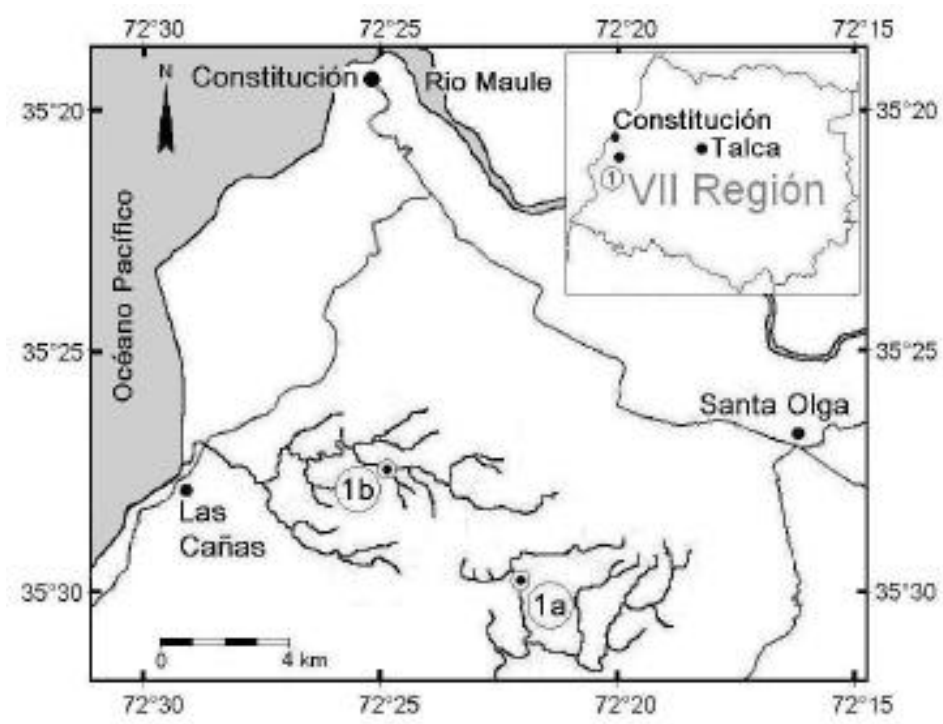

Figura1. Ubicación de las poblaciones de Hymenoglossum cruentum y Hymenophyllum darwinii en Quebrada Pellín (1a) y Trichomanes exsectum en quebrada Los Coigües (1b).

\section{AGRADECIMIENTOS}

Se agradece el apoyo a los Dres. José San Martín, Alejandro Troncoso y Patricio Peñailillo de la Universidad de Talca, al Dr. Roberto Rodríguez de la Universidad de Concepción y a Roberto L. Inzulza.

\section{BIBLIOGRAFIA}

Baeza, M., E. Barrera, J. Flores, C. Ramírez \& R. Rodríguez. 1998. Categorías de conservación de Pteridophytas nativas de Chile. Boletín del Museo Nacional de Historia Natural 47: 23- 46.

Diem, J. \& J. DE Lichtenstein 1959. Las Himenofiláceas del área argentino-chilena del sud. Darwiniana 11: 611-760.

Rodriguez, R. 1995. Pteridophyta. En: Flora de Chile, Vol. 1 (Eds. C. Marticorena \& R. Rodríguez), pp.
119-309. Ediciones Universidad de Concepción, Concepción.

Sepúlveda, C. \& A. Stoll. 2003. Presencia de Nothofagus alpina (Poepp. et Endl.) Oerst. (Fagaceae) en el borde costero de la Región del Maule, Chile central. Gayana Botanica 60: 134-135.

Sepúlveda, C. \& A. Troncoso. 2004. Hallazgo de Laureliopsis philippiana (Looser) Schodde (Atherospermataceae) en el área costera de la Región del Maule, Chile central. Gayana Botanica (en prensa).

Troncoso, A. \& J. SAn Martín, 1988. Ampliación de área para diversas especies de plantas vasculares en la Cordillera de la Costa de la Región del Maule. Boletín del Museo Nacional de Historia Natural 41: 45-56.

Villagrán, C., C. Le-quesne, J.C. Aravena, H. Jimenez \& F. Hinojosa. 1998. El rol de los cambios de clima del cuaternario en la distribución actual de la vegetación de Chile central-sur. Bamberger Geographische Schriften 15: 227-242.

Fecha de recepción: 29.03.04

Fecha de aceptación: 10.05.04 